Document downloaded from:

http://hdl.handle.net/10251/93334

This paper must be cited as:

Insa Franco, R.; Salvador Zuriaga, P.; Inarejos Mesa, J.; Roda Buch, A. (2012). Analysis of the influence of under sleeper pads on the railway vehicle/track dynamic interaction in transition zones. Proceedings of the Institution of Mechanical Engineers Part F Journal of Rail and Rapid Transit. 226(4):409-420. doi:10.1177/0954409711430174

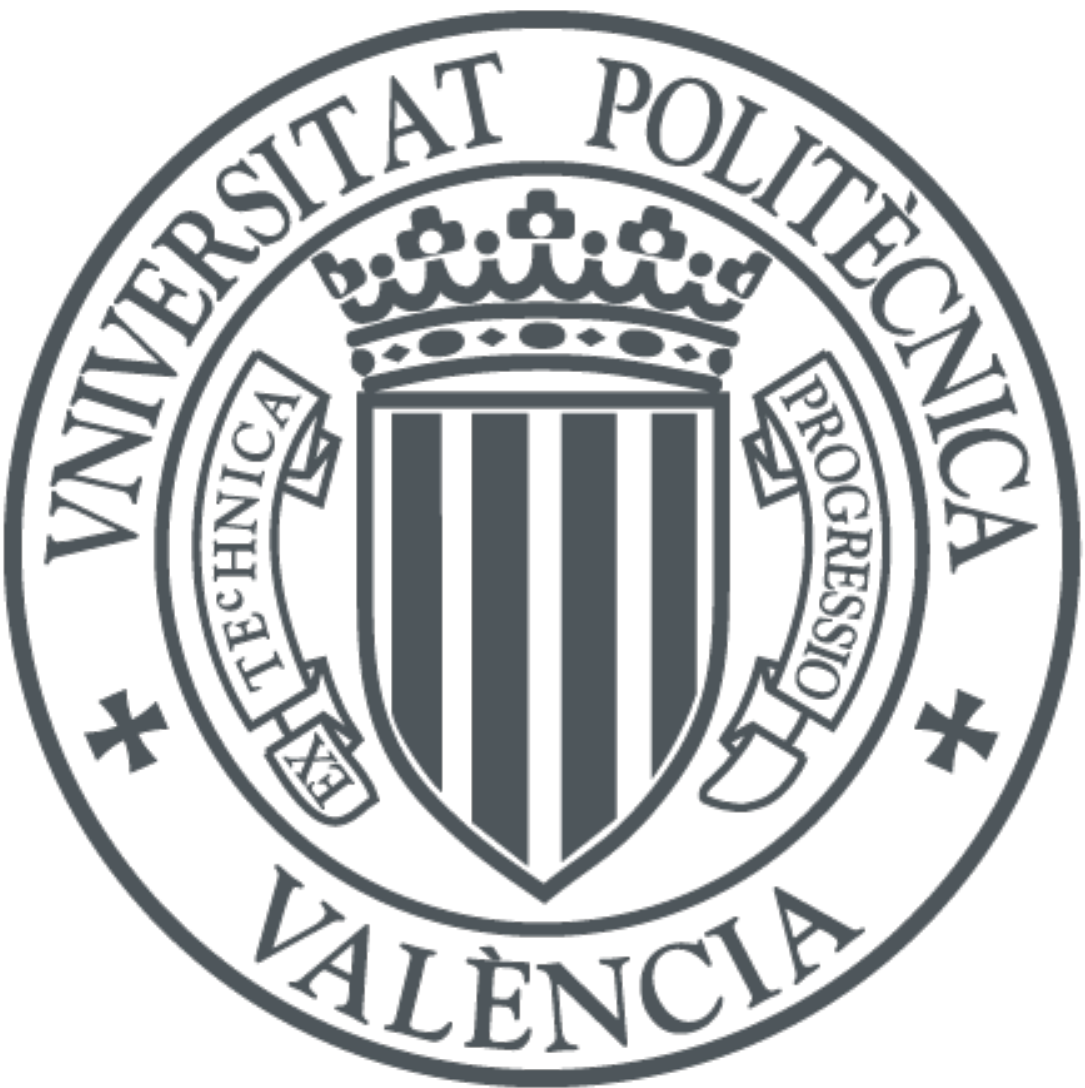

The final publication is available at

http://doi.org/10.1177/0954409711430174

Copyright SAGE PUBLICATIONS LTD

Additional Information 


\title{
Analysis of the influence of under sleeper pads on the railway vehicle/track dynamic interaction in transition zones
}

Ricardo Insa ${ }^{\mathrm{a}}$, Pablo Salvador ${ }^{\mathrm{a}, *}$, Javier Inarejos ${ }^{\mathrm{a}}$, Alejandro Roda ${ }^{\mathrm{b}}$

a Departamento de Ingeniería e Infraestructura de los Transportes, Universidad Politécnica de Valencia. Camino de Vera s/n, 46022 Valencia, Spain

b Centro de Investigación en Tecnología de Vehículos, Departamento de Ingeniería Mecánica y de Materiales, Universidad Politécnica de Valencia, Camino de Vera s/n, 46022 Valencia, Spain

\begin{abstract}
Sharp changes in track vertical stiffness may accelerate train and infrastructure deterioration and even raise the risk of derailment up to problematic values. This is due to the huge overloads and unloads which are repeatedly produced among the different track and vehicle components. The phenomenon has grown in importance as the operation velocities have been raised together with the expansion of high speed railways. In order to solve this problem, several technical solutions try to smooth the change in vertical stiffness along the track. In the present paper, the combination of transition regions and under sleeper pads has been studied. The research has been performed by means of a dynamic vehicle/track interaction model based on a modal substructuring approach. The analysed variables deal with different track configurations, train travelling speeds and the stiffness of the under sleeper pads. The results show the suitability of combining transition zones with under sleeper pads for achieving a more homogeneous vertical stiffness along the tracks that permits enhancing the dynamic behaviour of the vehicle/track system.
\end{abstract}

\section{Introduction}

\footnotetext{
* Corresponding author. Tel. +34 648231661

E-mail address: pabsalzu@cam.upv.es
} 
Together with the increase of the operational speed in railways, the topic of transition regions has grown in importance. The fact that embankments are less stiff than structures in a railway infrastructure produces settlements to be different -greater in the first case- for a given train load. This difference in settlements lowers the train stability which, in some cases where the travelling speed is high enough, i.e. High Speed Trains, may lead to a risk of derailment. Plus, the maintenance works carried out in these transition zones in order to keep their properties under acceptable service conditions increase enormously the overall operational costs. Thus, there is an evident need of coming up with a reliable solution if the speed of the trains wants to be raised, which is reflected throughout the literature and research focused on this topic.

Track transitions have been analysed either from the point of view of the infrastructure or from the point of view of the train behaviour, in which the wheel/rail contact force is included. Regarding the infrastructure, in Ref. [1] a finite element model whose most distinctive characteristic is its attempt to simulate the real behaviour of the diverse materials which make up the structure of the track in a transition was developed. The study considered the surface defining the embankment slope as free and took into account the elastoplastic behaviour of the soil. Hyslip et al. [2] also carried out a railway transition case study where the settlements of bridges approach produced by heavy freight trains have been observed. In addition, Smith et al. [3] presented a parametric study focused on the effects of train velocity and stiffness of embankment materials.

On the subject of wheel/rail contact force variation against stiffness changes, Lei and Mao [4] set up a dynamic model in which the vehicle and the track are considered as 
two different dynamic structures that are solved independently with an iterative scheme. Coupling the vehicle system and the railway track is realized through interaction forces between the wheels and the rail. The main conclusion is that the main source of the problem is the sudden permanent settlement of the track vertical profile in the track transition. Furthermore, $\mathrm{Li}$ and $\mathrm{Wu}$ [5] studied the wheel/rail impact produced when a train runs from a ballasted track and a floating slab track and vice versa in order to evaluate the actual necessity of building transition regions between both types of tracks. The conclusions of this research were that wheel/rail impact load is moderate for a light FST and increases with the vehicle speed or decreasing the natural frequency of the FST. A more global research regarding different situations involving changes of vertical stiffness is presented in [6], suggesting some solutions for smoothing them such as the use of under sleeper pads (USP) or grouting.

Respect to rail pads and USP, Johannson et al. [7] analysed the influence of this material on the dynamic train-track interaction in a parametric study. They consider the USP dynamic modulus, which is three times stiffer than the static modulus. These are the three main conclusions of this paper: the UPS only influences the lower part of the frequency spectrum $(<250 \mathrm{~Hz})$, the highest rail pad stiffness in combination with the lowest USP stiffness yields the highest vertical sleeper acceleration and the railseat loads are almost independent of the USP stiffness. Furthermore, no clear relationship between wheel-rail contact forces and USP stiffness was found.

The research presented in this paper aims at analysing the suitability of using transition regions between embankments and structures in a railway infrastructure and the possibility of combining them with under sleeper pads in order to enhance their effects. 
The analysis has been carried out from the point of view of train dynamics, for what special attention on the dynamic modelling of the tracks, the train and the wheel/rail contact has been paid to. Appropriate dynamic models can be found in [8], which investigates the effects on wheel/rail interaction due to the parametric excitation, and in [9] and [10], especially useful for simulating the dynamics of high speed trains running on nonlinear tracks. Dynamic models based on modal decomposition, with lower computational requirements, can be found in Nielsen and Igeland [11] and in Baeza et al. [10].

In the following pages, the adopted model is commented, emphasising the modelling of the different elements and materials. Then, the different situations in which the model has been applied are described, contemplating the use of USP with different stiffness and trains running at different speeds. The results are given in terms of wheel/rail contact force and relative overload and unload respect to the static load.

\section{The problem in the transition zones}

Changes in track vertical stiffness cause an increment of dynamic forces, whose magnitude is determined by the speed of the trains, the ratio between stiffnesses, the soil damping and the transition length. Furthermore, the differential settlements on tracks lead to a significant increment of accelerations in the vehicles, which cannot achieve the passengers' comfort levels or the maxima dynamic forces allowed by the tracks. Such magnification of vertical forces produces track deterioration and increases the infrastructure maintenance costs [12]. 
In order to reduce this damaging effect, transition regions or transition zones are built between sections with different stiffness, e.g. embankments and structures, as commented in Section 1. In this way, a smoother deformation is achieved through the different sections of the transition, so the increment of stiffness is progressively achieved before reaching the structure. The schematic solution of the transition zone proposed by the Spanish Railway Administrator ADIF [13] is shown in Fig. 1a, whereas a real transition zone embankment-bridge is shown in Fig. $1 \mathrm{~b}$.

It is also possible to change the most rigid zones, normally acting over the abutment or the deck of the bridge by setting up some elastic materials either between the sleepers and the ballast or between the ballast and the structure. Logically, a combination of the measures described above is suitable too.

From all the possible solutions, under sleeper pads have the advantage of contributing to a better redistribution of stresses in the lower layers of ballast tracks, by increasing the contact surface area, lowering transmitted stresses and thus preserving the ballast layer.

\section{Model description}

For this research, a dynamic model based on modal substructuration has been adopted, since it is less time consuming than a FEM model, in terms of computational cost, and still allows for considering some non-linearities among the different elements in the model. In this way, calculations have been performed following Ref. [11]. It consists in 
a hybrid model which combines both physical and modal coordinates and obtains the results through numerical integration. Only vertical dynamics are considered, but asymmetric behaviour respect to the track axis is permitted.

In this way, the model divides the whole system into three kinds of elements: the rails, the sleepers and the vehicle. Non-linearities are taken into account in the linkage among the previous elements, i.e. the wheel/rail contact, the rail pads and the ballast. Regarding the stiffness for the ballast, the subballast and the USP, the Winkler theory has been assumed.

The global coordinate system $x y z$ is defined with the positive $x$-axis parallel to the rail in the direction of the vehicle motion. The $y$-axis is transverse to the track and the vertical z-axis is positive upwards. Fig. 2 shows the model with the different degrees of freedom, the forces acting among the different elements which compose the model, i.e, the vehicle, the rails and the sleepers, and the reference axis.

\subsection{Rails and sleepers}

The rails and the sleepers have been considered as Timoshenko beams, whose modal properties can be obtained by taking separately each element. Using the modal substructuring approach, the physical displacements in the sleepers are related with their modal coordinates and the forces transmitted through the railpads to modal forces through the following expressions:

$u_{s}(y, t)=\sum_{n=-1}^{N_{m s}} \psi_{n}(y) q_{s n}^{S}(t), f_{s n}^{S}(t)=\sum_{r=1}^{2} F_{r s}^{p} \psi_{n}\left(d_{r}\right)$, 


\section{(1)}

where $u_{s}(y, t)$ represent the vertical displacements of sleeper $s ; \psi_{n}(y)$ is the mode shape of the $n^{\text {th }}$ non-damped mode, which is mass-normalised and is associated to the natural angular frequency $\omega_{n} ; q_{s n}^{S}(t)$ and $f_{s n}^{S}(t)$ represent the modal coordinate and the modal force associated to mode $n$ of sleeper $s$, respectively; $F_{r s}^{p}$. are the forces exerted by the rails through the railpads applied on the rail seats in $y=d_{r}$ (index $r=1,2$ corresponds to the right and left rail, respectively). Mode numbers $n=-1$ and 0 are reserved for modes which do not produce elastic strain in the beam and modal truncation is performed considering a finite number $N_{m s}$ of sleeper modes.

Similarly, the modal transformations for the rails are:

$v_{r}(x, t)=\sum_{m=-1}^{N_{m r}} \phi_{m}(x) q_{r m}^{R}(t), f_{r m}^{R}(t)=\sum_{a=1}^{N_{a x}} F_{r a}^{c} \phi_{m}\left(c_{a}+V t\right)-\sum_{s=1}^{N_{s}} F_{r s}^{p} \phi_{m}\left(b_{s}\right)$,

where $v_{r}(x, t)$ represent the vertical displacements of rail $r ; \phi_{m}(x)$ is the mode shape of the $m^{\text {th }}$ non-damped mode, which is mass-normalised and is associated to the natural angular frequency $\lambda_{m} ; q_{r m}^{R}(t)$ and $f_{r m}^{R}(t)$ represent the modal coordinate and the modal force associated to mode $m$ of rail $r$, respectively; The position of each wheelset is $x=$ $c_{a}+V t$, where $V$ is the vehicle speed, $c_{a}$ is the corresponding initial coordinate and subindex $a$ is an integer which numbers the wheelsets. Each rail $r$ is acted upon by a set of forces $F_{r s}^{p}$ exerted by the sleepers through the railpads, located in rail section $x=b_{s}$, and another set of forces $F_{r a}^{c}$ transmitted by the wheels through the wheel-rail contacts. Mode numbers $n=-1$ and 0 are reserved for modes which do not produce elastic strain in the beam and modal truncation is performed considering a finite number $N_{m r}$ of rail modes. Eq. (2) have been obtained by considering the rails as a Timoshenko beam with 
free-free boundary conditions, so the corresponding mode shapes and natural frequencies can be determined analytically upon Ref. [14].

Once the modal coordinates and the modal forces have been introduced, the model equation, for the case of mode $n$ of sleeper $s$ is represented by the following set of equations:

$\dot{q}_{s n}^{S}(t)=-2 \xi_{n} \omega_{n} q_{s n}^{S}(t)+p_{s n}^{S}(t), \quad \dot{p}_{s n}^{S}(t)=-\omega_{n}^{2} q_{s n}^{S}(t)+f_{s n}^{S}(t)$,

where coordinate $p_{s n}^{S}(t)$ has been introduced along with coordinate $q_{s n}^{S}(t)$ in order to obtain a base of the phase space for the sleeper dynamics and $\xi_{n}$ stands up for considering the damping effect associated to each sleeper mode ( $s$ varies from 1 to the number of sleepers $N_{s}$, and $n$ from -1 to the number of sleeper modes $N_{m s}$ ).

Analogously, the equations of motion for the mode $m$ of rail $r$ is defined by

$\dot{q}_{r m}^{R}(t)=-2 \zeta_{m} \lambda_{m} q_{r m}^{R}(t)+p_{r m}^{R}(t), \quad \dot{p}_{r m}^{R}(t)=-\lambda_{m}{ }^{2} q_{r m}^{R}(t)+f_{r m}^{R}(t)$,

where coordinate $p_{r m}^{R}(t)$ has been introduced along with coordinate $q_{r m}^{R}(t)$ in order to obtain a base of the phase space for the sleeper dynamics and $\zeta_{m}$ stands up for considering the damping effect associated to each sleeper mode ( $r$ varies from 1 to the number of rails $N_{r}$, and $m$ from -1 to the number of rail modes $N_{m r}$ ).

\subsection{Vehicle model}

The railway vehicle is modelled as a multibody system where the wheelsets, bogie frames and carbody are considered as rigid bodies joined by means of linear suspensions (see Fig. 2), so the equations which explain their dynamics assuming small displacements are: 


$$
M \ddot{w}+\mathrm{D} \dot{w}+\mathrm{Kw}=\mathrm{F}_{\mathrm{ext}}+\mathrm{F}_{\mathrm{c}}
$$

where $\mathbf{w}$ are the coordinates for a lumped mass model of the vehicle; $\mathbf{M}, \mathbf{D}$ and $\mathbf{K}$ are, respectively, the mass, viscous damping and stiffness matrices; $\mathbf{F}_{\text {ext }}$ is the vector of the external forces to the vehicle, mainly composed of gravitational forces and $\mathbf{F}_{\mathrm{c}}$ is the vector of the wheel/rail contact forces. In this way, for the $a^{\text {th }}$ wheelset, two independent displacements are studied corresponding to vertical displacement $z_{a}(t)$ and roll angle $\theta_{a}(t)$. On each wheelset $a$, forces $F_{r a}^{c}(r=1,2)$ from each rail are applied through the wheel-rail contacts.

\subsection{Nonlinear forces}

Nonlinear behaviour is mainly observed in the elements which interconnect the different subsystems. Such elements are the wheel-rail contact, the rail pads and the ballast. Non linear forces derived from wheel-rail contact and railpads have been considered in the model. Wheel-rail interaction is modelled by the contact force $F_{r a}^{c}$. In general, this force is expressed as a function of the relative displacement between wheel and rail at the contact point, and it depends on the non-deformed wheel-rail geometry and the elastic characteristics of the wheel-rail contact. For this case, since the wheels are assumed to have a perfect round shape, the Hertzian model has been adopted, as several authors do $[4,5,7,10,11]$. It has the following expression:

$F^{c}=k_{H} \delta^{1.5}$

being $k_{H}$ a constant which depends on contact geometry and the mechanical features of the materials, and $\delta$ the approach of the contact points of the two elastic bodies in contact. 
The stiffness and damping properties of the railpad determine the force $F_{r s}^{p}$ transmitted between rails and sleepers. The force is a function of the relative displacement $\chi$ of the elements which are joined by the railpad, and its time derivative $\dot{\chi}$ according to

$F_{r s}^{p}=k_{p} \chi+c_{p} \dot{\chi}+h_{p}(\chi, \dot{\chi})$

where the nonlinearities associated to railpad behaviour are considered in $h_{p}(\chi, \dot{\chi})$.

\subsection{Numerical resolution}

The sets of differential equations given in (3) and (4) for each sleeper and each rail, respectively, and in (5) for the vehicle are linked through the formulas (6) and (7) associated to the forces transmitted between the substructures. They are solved by means of a standard numerical procedure such as Runge-Kutta implemented in a program from a NAG library. The suitability of this technique lies on the low computational cost, since only a few number of coordinates is considered, and on the simplicity when introducing complex models of wheel/rail contact, rail pads or ballast.

\section{Results and discussion}

The simulation performed corresponds to the train entering the structure from the embankment. The study has been focused on the train travelling on this direction rather than analysing both directions since the highest wheel/rail impacts are produced when a train runs from a soft track configuration to a stiffer one $[1,4,5,6]$. A total of 80 sleeper bays have been considered, from where 15 of them are placed in the structure. The track properties are shown in Table 1 and correspond to those adopted for the High Speed 
Line between Valencia and Játiva (Spain). The train properties are shown in Table 1 as well and correspond to a train Talgo Series 350 locomotive.

A schematic view of the system is shown in Fig. 3, where the transition zone consists of the embankment, a granular soil and a hydraulic soil. In this sense, the transition zone, which includes the granular soil and the hydraulic soil, begins in the $35^{\text {th }}$ sleeper and in the $66^{\text {th }}$ sleeper so does the structure. Together with the infrastructure, the variation along the track of sleeper apparent vertical stiffness for the case of building a transition zone and placing USP only under the bridge sleepers is represented. It can be appreciated how the transition zone smoothes the change of the vertical stiffness before reaching the structure. The USP highly contribute to the stiffness change reduction by lowering the whole vertical stiffness of the track placed on the bridge.

Two main groups of results have been obtained from the train-track-bridge dynamic system previously described. In the first group of results, different simulations have been performed for speeds of 100,150, 200, 150 and $300 \mathrm{~km} / \mathrm{h}$, where the following configuration have been compared:

- $\quad$ There is no transition zone (TZ) nor USP

- $\quad$ There is transition zone but there are no USP

- $\quad$ There is transition zone and $100 \mathrm{kN} / \mathrm{mm}$ stiffness USP in the structure

- $\quad$ There is transition zone and $200 \mathrm{kN} / \mathrm{mm}$ stiffness USP in the structure

The results correspond to the first axle contact force in the running direction and are presented in Fig. 4, Fig. 5 and Fig. 6 for the speeds of 100, 200 and $300 \mathrm{~km} / \mathrm{h}$, respectively. The wheel/rail contact force is represented in the $y$-axis, whereas the 
position of the axle along the track is shown in the $x$-axis in terms of the sleeper number. Along the embankment, the force has a regular periodic shape whose response coincides with the sleeper cadence, being associated to the parametric excitation of the track. Small variations appear as the transition zone begins, which become higher when the axles pass over the beginning of the structure. From there, the maximum and minimum values for the vertical force transmitted between the wheel and the rail can be appreciated. These values can be utilised for calculating the vehicle rolling safety and its degree of harm respect to the tracks upon UIC 518 [15]. The worst case appears for a vehicle velocity of $300 \mathrm{~km} / \mathrm{h}$ entering the bridge when no transition zone nor USP are used.

From the second group of results, the maximum unload values for speeds of 100, 150, 200, 250 and $300 \mathrm{~km} / \mathrm{h}$ are shown in Figs. 7 and 8, where the following track configurations have been considered:

- $\quad$ USP have been placed only in the structure with stiffness varying from 50 to 200 $\mathrm{kN} / \mathrm{mm}$ (no transition zones)

- There exists transition zone and USP placed only in the structure with stiffness varying from 50 to $200 \mathrm{kN} / \mathrm{mm}$

- USP have been placed both in the embankment and the infrastructure with stiffness varying from 50 to $200 \mathrm{kN} / \mathrm{mm}$ (no transition zones)

- There exists transition zone and USP placed both in the embankment and the structure with stiffness varying from 50 to $200 \mathrm{kN} / \mathrm{mm}$

The influence of the USP's stiffness can be appreciated in Fig. 7. The main characteristics of the results are: the unloading grows for all cases with vehicle speed; 
all cases show a minimum in the percentage unload between 100 and $150 \mathrm{~km} / \mathrm{h}$; the lowest unload values are generally achieved with the less stiff USP when it is placed only in the structure. For the case of overloads, analogue results are obtained.

The influence of the different track designs can be appreciated in Fig. 8, where the maximum unload values are shown for all the cases with a USP stiffness of 100 $\mathrm{kN} / \mathrm{mm}$. It can be seen that the best rolling performance is achieved when using transition zones and placing under sleeper pads only in the structure. In terms of USP stiffness, those with the lowest stiffness, together with the use of transition zones produce the best dynamic behaviour.

Some other results can be found from the first set of results. The influence of the travelling speed can be appreciated in Fig. 9. In this figure, the maximum overload and unload respect to the static load is shown for different running speeds and the different track configurations. The relative overload increases with the velocity for all track configurations, but a relative maximum around $150 \mathrm{~km} / \mathrm{h}$ can be appreciated, which reaches a value of 1.35 for those cases when USP are not installed. The effect the USP introduce is a reduction of the global vertical stiffness and an increase of the damping coefficient, thus lowering the peak value and displacing this peak to lower running speeds. This phenomenon is well known in other train/track dynamic interaction problems, such as unbalanced axles or flat wheels. Its causes are complex and occur due to dynamic amplifications of the system, whose prediction is not easy since they cannot be related to resonance problems. Something analogue occurs with the relative unloads, for which minimum relative unload values of 0.65 appear when no using USP. 
For speeds higher than $200 \mathrm{~km} / \mathrm{h}$, the relative overload increases. This increment is especially important for the case without building a transition zone and without placing USP, when for speeds of $300 \mathrm{~km} / \mathrm{h}$ an increment of $25 \%$ is reached. If a transition zone is built, the overloads increments are reduced down to the $10 \%$ of the static load. The overloads are further reduced when, together with transition regions, USP are placed under the sleepers on the structure. Therefore, the combination of transition zones with USP may provide a sufficient smooth change of vertical stiffness for high speeds up to $300-350 \mathrm{~km} / \mathrm{h}$. Again, for the case of relative unloads, the analogue situation is presented.

The same effect of Fig. 9 is shown in Fig. 10 in terms of rail contact point vertical acceleration when building a transition zone and placing USP of $200 \mathrm{kN} / \mathrm{mm}$ only under the sleepers located on the structure. From this figure, the dynamic amplification around $V=120 \mathrm{~km} / \mathrm{h}$ is observed, as well as the oscillation movement due to the parametric excitation of the track, which is progressively amplified as the train enters the transition zone and the structure due to the stiffness change.

\section{Conclusions}

The suitability of placing under sleeper pads in combination with transition regions in order to minimize dynamic strains in the wheel/rail interaction has been analysed throughout this paper. For this purpose, a dynamic wheel/rail interaction model based on a modal substructuring approach has been employed and different track configurations, involving USP with different stiffnesses and their location have been 
evaluated. Results have been given in terms of wheel/rail contact force and unload percentage respect to the static value.

Upon the obtained results, it is suitable to combine under sleeper pads with transition regions. As a matter of fact, track configurations without USP may be not appropriate, especially for speeds higher than $250 \mathrm{~km} / \mathrm{h}$, and by lowering the vertical stiffness under the sleeper, a more homogeneous track is achieved and the dynamic effects associated to the stiffness discontinuity are diminished. The most favourable configuration is those with a transition region built with granular soil and hydraulic soil in conjunction with USP placed only in the structure. This is, though, slightly better than the solution of placing USP under all the sleepers, which can be more suitable from other points of view such as ballast preservation.

These conclusions are limited by the fact that the mechanical properties of the pads cannot be achieved due to that they are discretely supported by the ballast. Moreover, the scope of the present analysis does not consider geometrical track defects such as the loss of vertical alignment caused by embankment settlements, the existence of a hanging sleeper and some other defect which may affect the dynamic response of the system.

\section{References}

1 Gallego, I. and López Pita, A. Numerical simulation of embankmentstructuretransition design. Proceedings of the Institution of Mechanical Enginners. Part F: Journal of Rail and Rapid Transit, 2009, 223(4), 13.

2 Hyslip, J.P., Li, D. and McDaniel, C.R. Railway bridge transition case study. Bearing Capacity of Roads, Railways and Airfields, Vols 1 and 2, 2009, 1341-1348.

3 Smith, M.E., Bengtsson, P.E. and Holm, G. Three-dimensional analyses of transition zones at railway bridges. In Schweiger, H.F., ed. Numerical Methods in Geotechnical Engineering, pp. 237-2422006). 
4 Lei, X. and Mao, L. Dynamic response analyses of vehicle and track coupled system on track transition of conventional high speed railway. Journal of Sound and Vibration, 2004, 271(3-5), 1133-1146.

5 Li, Z.G. and Wu, T.X. On vehicle/track impact at connection between a floating slab and ballasted track and floating slab track's effectiveness of force isolation. Vehicle System Dynamics, 2009, 47(5), 19.

6 Dahlberg, T. Railway Track Stiffness Variations - Consequences and Countermeasures. International Journal of Civil Engineering, 2010, 8(1), 1-12.

7 Johansson, A., Nielsen, J.C.O., Bolmsvik, R., Karlström, A. and Lundén, R. Under sleeper pads--Influence on dynamic train-track interaction. Wear, 2008, 265(910), 1479-1487.

8 Wu, T.X. and Thompson, D.J. On the parametric excitation of the wheel/track system. Journal of Sound and Vibration, 2004, 278(4-5), 725-747.

9 Baeza, L. and Ouyang, H. A railway track dynamics model based on modal substructuring and a cyclic boundary condition. Journal of Sound and Vibration, 2011, 330(1), 75-86.

10 Baeza, L., Roda, A. and Nielsen, J.C.O. Railway vehicle/track interaction analysis using a modal substructuring approach. Journal of Sound and Vibration, 2006, 293(1-2), 112-124.

11 Nielsen, J.C.O. and Igeland, A. VERTICAL DYNAMIC INTERACTION BETWEEN TRAIN AND TRACK INFLUENCE OF WHEEL AND TRACK IMPERFECTIONS. Journal of Sound and Vibration, 1995, 187(5), 825-839.

12 Esveld, C. Modern railway track. (MRT, Zaltbommel, 2001).

13 Instrucciones Generales para los Proyectos de Plataforma IGP. (Adif, Madrid, 2008).

14 Weaver, W., Timoshenko, S.P. and Young, D.H. Vibration Problems in Engineering. (Willey, New York, 1990).

15 UIC-518. Testing And Approval Of Railway Vehicles From The Point Of View Of Their Dynamic Behaviour - Safety - Track Fatigue - Ride Quality. 2009. 


\section{Figure captions}

1. Fig. 1a. Schematic profile of a embankment-bridge transition zone proposed by ADIF

Fig. 1b. Transition zone built between an embankment and a bridge in the MadridValencia High Speed Line

2. Fig. 2. Substructuring model, showing the force interactions among the different elements

3. Fig. 3. Variation along track of sleeper apparent stiffness due to transition zone and USP under bridge sleepers

4. Fig. 4. Wheel/rail contact force response. Train speed $100 \mathrm{~km} / \mathrm{h}$

5. Fig. 5. Wheel/rail contact force response. Train speed $200 \mathrm{~km} / \mathrm{h}$

6. Fig. 6. Wheel/rail contact force response. Train speed $300 \mathrm{~km} / \mathrm{h}$

7. Fig. 7. Minimum wheel/rail contact force value depending on the speed of the train for different track designs: a) no transition region, USP in the structure; b) transition region, USP in the structure; c) no transition region, USP under all the sleepers; d) transition region, USP under all the sleepers

8. Fig. 8. Minimum wheel/rail contact force value in terms of unload percentage, depending on the speed of the train for different track designs. USP stiffness 100 $\mathrm{kN} / \mathrm{mm}$

9. Fig. 9. Comparison $F_{c} / F_{0}$ vs. Velocity. USP only in the structure when placed

10. Fig. 10. Rail contact point acceleration with transition zone and USP $200 \mathrm{kN} / \mathrm{mm}$

\section{Table Captions}

1. Table 1. Numeric values for the data input of the model 